\title{
Elastoplasticity Mediates Dynamical Heterogeneity Below the Mode Coupling Temperature
}

\author{
Rahul N. Chacko®, ${ }^{1,2, *}$ François P. Landes $\odot,{ }^{3}$ Giulio Biroli, ${ }^{4}$ Olivier Dauchot $\odot,{ }^{5}$ \\ Andrea J. Liu $\odot{ }^{1}{ }^{1}$ and David R. Reichman ${ }^{6}$ \\ ${ }^{1}$ Department of Physics and Astronomy, University of Pennsylvania, Philadelphia, Pennsylvania 19104, USA \\ ${ }^{2}$ James Franck Institute, University of Chicago, Chicago, Illinois 60637, USA \\ ${ }^{3}$ Université Paris-Saclay, CNRS, Laboratoire Interdisciplinaire des Sciences du Numérique, Orsay 91400, France \\ ${ }^{4}$ Laboratoire de Physique de l'École normale supérieure, ENS, Université PSL, CNRS, Sorbonne Université, \\ Université de Paris F-75005 Paris, France \\ ${ }^{5}$ UMR Gulliver 7083 CNRS, ESPCI, PSL Research University, 10 rue Vauquelin, Paris 75005, France \\ ${ }^{6}$ Department of Chemistry, Columbia University, 3000 Broadway, New York, New York 10027, USA
}

(Received 4 March 2021; revised 14 May 2021; accepted 10 June 2021; published 22 July 2021)

\begin{abstract}
As liquids approach the glass transition temperature, dynamical heterogeneity emerges as a crucial universal feature of their behavior. Dynamic facilitation, where local motion triggers further motion nearby, plays a major role in this phenomenon. Here we show that long-ranged, elastically mediated facilitation appears below the mode coupling temperature, adding to the short-range component present at all temperatures. Our results suggest deep connections between the supercooled liquid and glass states, and pave the way for a deeper understanding of dynamical heterogeneity in glassy systems.
\end{abstract}

DOI: 10.1103/PhysRevLett.127.048002

Dynamic facilitation is the process whereby relaxation of a local region in a glassy system enables another local region to subsequently move and relax. Facilitation plays a major role in the spatiotemporal pattern of correlated relaxation events characteristic of supercooled liquids, referred to as "dynamical heterogeneity," which is perhaps the most striking hallmark of glassy dynamics [1]. The underlying structural and microscopic origins of dynamic facilitation (also called kinetic facilitation) and dynamical heterogeneity are still widely debated. At high temperatures facilitation appears to be a local, short-ranged process [2-6]. However it has been appreciated for decades that some relaxation processes in glassy media involve longranged correlations. Given that dynamic facilitation in supercooled liquids arises initially at short timescales, where the system should behave effectively as a solid, it is natural to wonder whether long-ranged elastic processes may influence facilitation and dynamical heterogeneity.

An extreme example of the influence of elasticity is the low-temperature relaxation of tunneling defects in glasses, which is mediated by long-ranged (dipolar) phonon exchange [7-9]. Another example is provided by the plastic behavior of amorphous solids. Local plastic deformations

Published by the American Physical Society under the terms of the Creative Commons Attribution 4.0 International license. Further distribution of this work must maintain attribution to the author(s) and the published article's title, journal citation, and DOI. in elastic media induce signature quadrupolar perturbations to the stress and strain fields in the surrounding material which decay as power laws in space [10]. The role of such fields in triggering relaxation elsewhere when an amorphous solid is mechanically deformed has been the focus of intense research activity during the last decade [11-17]. This triggering by stress is a type of "elastically mediated" dynamic facilitation that has been thoroughly studied in the context of the rheology of amorphous solids by means of elastoplastic models [16], in which each rearrangement perturbs the stress of the surrounding material, thereby triggering new rearrangements when the perturbing stress surpasses some local threshold value. It should be noted, however, that elastoplastic models do not incorporate the type of thermal motion that occurs in equilibrium liquids. Recent reports of anisotropic spatial correlations in stress or strain in liquids [18-23] have suggested that elasticity might play a role even at high temperatures, but it is now understood that such stress correlations must arise for isotropic systems in mechanical equilibrium and do not require elasticity [24,25].

Here we study by numerical simulation the effects of elasticity on dynamic facilitation in supercooled liquids at temperatures above and below the mode coupling temperature, $T_{\mathrm{MCT}}$. We examine both the strain response to a rearranging particle as well as the pair correlation function of rearrangements, $g_{\text {hop }}(\boldsymbol{r})$, characterizing the probability of finding a rearrangement at a relative position $\boldsymbol{r}$ within a short time interval following a rearrangement at the origin. At all temperatures studied we find that the strain response 
of the neighborhood of a rearranging particle is anisotropic and qualitatively consistent with the response of a linearly elastic medium. The pair correlation function of rearrangements, however, reveals a change of behavior with temperature. At high temperatures, dynamic facilitation is local; i.e., it acts only on nearby regions, as is indeed assumed in simple models [3,26]. However, dynamic facilitation becomes progressively longer-ranged and elastically mediated with decreasing temperature. Remarkably, the emergence of long-ranged facilitation takes place near $T_{\mathrm{MCT}}$, strengthening the interpretation of the mode coupling crossover as the temperature at which the system starts to display solidlike behaviors $[17,27,28]$.

We conduct microcanonical molecular dynamics simulations, using LAMMPS [29], of a $d=2$ dimensional polydisperse system of particles first introduced in [30]. The swap Monte Carlo algorithm [31,32] allows for the equilibration of this system even well below the mode coupling theory temperature $T_{\mathrm{MCT}}$ [30]. The particles have random diameters $\sigma \in\left[\sigma_{\min }, \sigma_{\max }\right]$ with probability density function $\propto \sigma^{-3}$, where $\sigma_{\min }$ and $\sigma_{\max }$ are determined by the mean $\bar{\sigma}$ and coefficient of variation $c_{\sigma}$ of $\sigma$. Particles interact via a pair potential $V(\tilde{r})=G \Theta\left(\tilde{r}_{0}-\tilde{r}\right)\left[\tilde{r}^{-12}+c_{0}+\right.$ $\left.c_{2} \tilde{r}^{2}+c_{4} \tilde{r}^{4}\right]$. Here, $\Theta$ is the Heaviside step function and $c_{0}$, $c_{2}$, and $c_{4}$ are chosen such that $V\left(\tilde{r}_{0}\right)=0$ and $V^{\prime}(\tilde{r})$ is continuously differentiable. The argument $\tilde{r}=r / \tilde{\sigma}$ is the interparticle separation $r$ between particles $i$ and $j$ with diameters $\sigma_{i}$ and $\sigma_{j}$ normalized by $\tilde{\sigma}=\frac{1}{2}\left(\sigma_{i}+\sigma_{j}\right)$ $\left(1-\epsilon\left|\sigma_{i}-\sigma_{j}\right|\right)$. Following [30], we choose $\tilde{r}_{0}=1.25$, $\epsilon=0.2$, and $c_{\sigma}=0.23$. We set $G, \bar{\sigma}$, the (uniform) particle mass $m$, and the Boltzmann constant $k_{B}$ to unity, thus defining our energy, length, mass, and temperature units. We study $N=10^{4}$ particles in an $L \times L$ periodic square box of side length $L=100$. For this system the onset temperature is $T_{0} \approx 0.236$ and the mode coupling temperature is $T_{\mathrm{MCT}} \approx 0.116$ [30].

We study rearrangements in 400 trajectories with independent initial velocities chosen from the Boltzmann distribution for each of 1000 separate equilibrium configurations for temperature $T=0.100,400$ configurations for $T=0.105$, and 202 configurations for $T=0.110,0.115$, $0.120,0.130,0.140$, and 0.150 , doubling our statistics by averaging over trajectories in both directions in time [33]. We follow equilibrium trajectories of the system, identifying rearranging particles as those with squared displacement $\Delta r^{2}>5 a^{2}$ between inherent structure (i.e., quenched) positions at times $t=0$ and $t=10^{2}[20,33]$. Here, $a^{2}$ is the (unquenched) mean squared displacement plateau height, a quantity that depends on the particle diameter and on the system temperature [33]. The timescale $\Delta t=10^{2}$ matches the time at which dynamic facilitation is at play, identified as the one at which individual rearrangements cluster together in space [33]. For reference, the ballistic time is $\tau_{\text {ballistic }} \approx 10^{0}$ in these units, and the relaxation time is $\tau_{\alpha}=3 \times 10^{2}$ and $\tau_{\alpha}=2 \times 10^{6}$ at the highest and lowest temperatures, respectively. For further characterization and details on the choice of $\Delta t$, see the Supplemental Material [33].

Our first observation is that the rearrangement of a given particle causes elasticlike displacements of other particles; i.e., the system, despite being a liquid, displays a solidlike response at short times. To show this, we proceed as follows. For each particle $i$ in the system, we identify the local best-fit linear strain tensor $\boldsymbol{E}_{i}[19,33,43]$ for quenched displacements in the neighborhood of the particle. We then extract strain invariants, such as the isotropic strain $\gamma_{\text {iso }, i}=\operatorname{Tr} \boldsymbol{E}_{i}$ and the deviatoric strain $\gamma_{\mathrm{dev}, i}=\sqrt{2 \operatorname{Tr} \boldsymbol{E}_{i}^{\prime 2}}$ (where $\left.\boldsymbol{E}_{i}^{\prime} \equiv \boldsymbol{E}_{i}-(1 / d) \operatorname{Tr} \boldsymbol{E}_{i} \boldsymbol{I}\right)$. Crucially, for rearranging particles, $\boldsymbol{E}_{i}$ also gives us local extensional and compressional axes from its orthonormal eigenvectors: we denote as $\boldsymbol{e}_{\mathrm{ext}, i}\left(\boldsymbol{e}_{\mathrm{com}, i}\right)$ the eigenvector corresponding to the largest (smallest) eigenvalue of $\boldsymbol{E}_{i}$. We then average over neighborhoods of rearranging particles translated such that the rearranging particle is at the origin at the start of the rearrangement interval (of duration $\Delta t$ ) and rotated such that the extensional axis of its local strain tensor is $\boldsymbol{e}_{\mathrm{ext}}=(1,0)$. From this we obtain a tensor field $\boldsymbol{E}(\boldsymbol{r})$ for the mean strain tensor $\boldsymbol{E}$ at position vector $\boldsymbol{r}$ relative to a rearranging particle at the origin with a horizontal local extensional axis. We also calculate the strain in this direction, $\gamma_{\mathrm{ext}} \equiv \boldsymbol{E}^{\prime}: \boldsymbol{e}_{\mathrm{ext}} \otimes \boldsymbol{e}_{\mathrm{ext}}$, where $\boldsymbol{e}_{\mathrm{ext}}$ and $\boldsymbol{e}_{\mathrm{com}}$ are eigenvectors of the local strain tensor $\boldsymbol{E}$ computed at the rearranging particle $i$, whereas $\boldsymbol{E}^{\prime}$ is the local deviatoric strain tensor of the neighbors $j$ of $i$. The dipolarity of $\gamma_{\text {iso }}$, quadrupolarity of $\gamma_{\mathrm{ext}}$, and the isotropic behavior of $\gamma_{\mathrm{dev}}$, shown in Fig. 1 for temperatures $T=0.100$ (left) and $T=0.150$ (right) spanning the full temperature range of our study, are the expected signatures of the response of an elastic solid [44].

We now assess the temperature dependence of the range of the elasticlike response by studying the angular Fourier series coefficients, which characterize the strength of the observed symmetries reported above [33]. More specifically, we consider the zeroth and second order coefficients $\hat{\gamma}_{\text {iso }, 0}$ and $\hat{\gamma}_{\text {iso }, 2}$, the zeroth order coefficient $\hat{\gamma}_{\mathrm{dev}, 0}$ and the fourth order coefficient $\hat{\gamma}_{\text {ext, } 4}$. As shown in Fig. $2, \hat{\gamma}_{\text {iso }, 2}$ and $\hat{\gamma}_{\text {ext, } 4}$ [Figs. 2(c), 2(d)] decay for $T \leq 0.12$ as $r^{-2}$ from $r=3$ up to a cutoff that increases slightly with decreasing $T$ but is roughly $r \simeq 10$. This power-law decay is expected for an elastic response [44]. The radial dependence of $\hat{\gamma}_{\mathrm{dev}, 0}$ [Fig. 2 (b)] deviates from the elastic response behavior at higher temperatures but appears to approach the expected behavior (dashed line) with decreasing temperature. This can be explained by the decreasing density of rearrangements per unit time with decreasing $T$, which allows the elastic displacement field from rearrangements to extend further into the material without being obscured by other rearrangements [33]. Finally, for the zeroth mode of $\gamma_{\text {iso }}$ [Fig. 2(a)], 


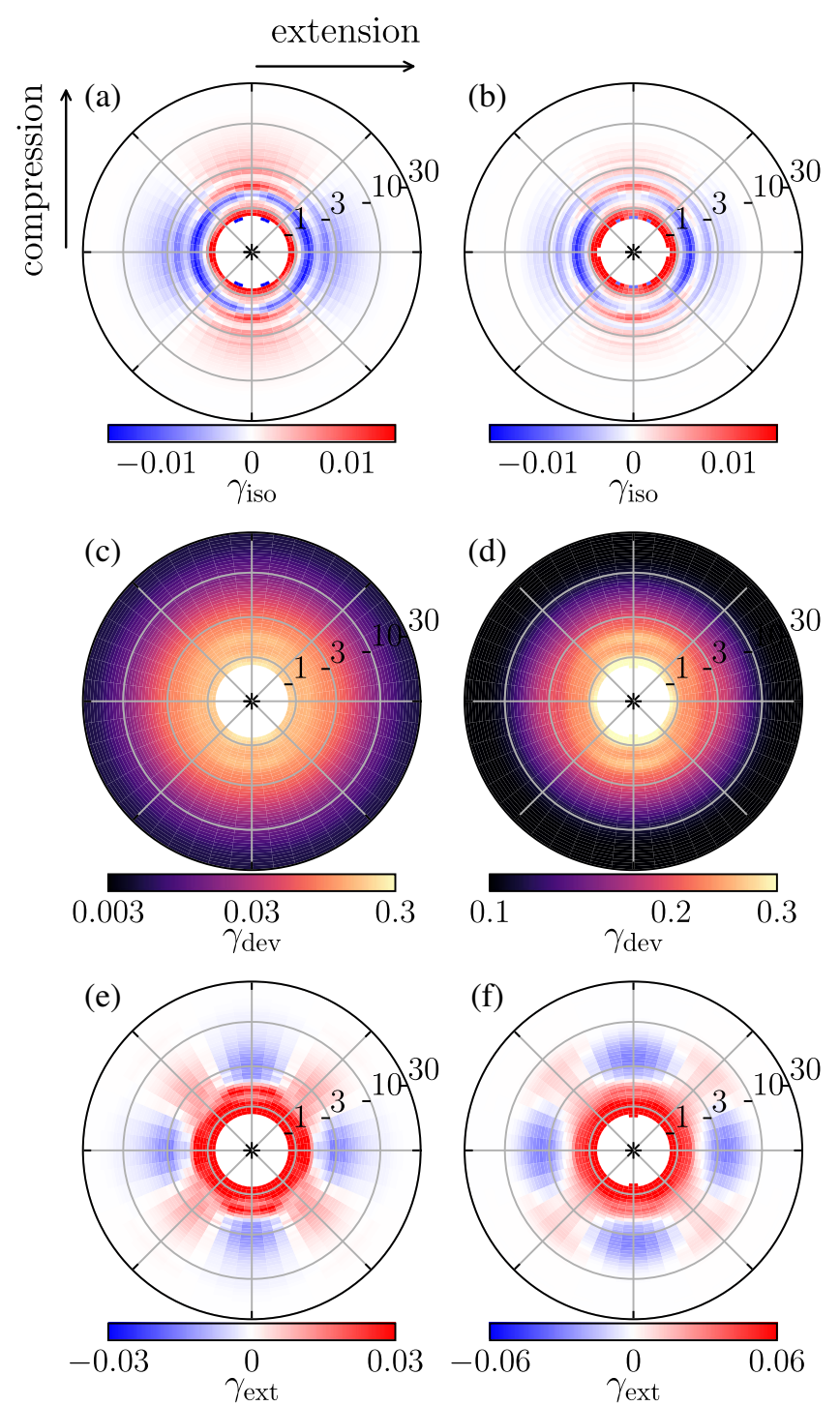

FIG. 1. Polar plots of (a),(b) $\gamma_{\text {iso }}=\operatorname{Tr} \boldsymbol{E}$, (c), (d) $\gamma_{\text {dev }}=\sqrt{2 \operatorname{Tr} \boldsymbol{E}^{\prime 2}}$, and (e),(f) $\gamma_{\mathrm{ext}}=\boldsymbol{E}^{\prime}: \boldsymbol{e}_{\mathrm{ext}} \otimes \boldsymbol{e}_{\mathrm{ext}}$ across a rearrangement interval $\Delta t=10^{2}$ as a function of the position $\boldsymbol{r}$ relative to a rearranging particle that was at $\boldsymbol{r}=\mathbf{0}$ at the start of the rearrangement interval for systems at temperatures $T=0.100$ (a),(c),(e) and $T=0.150$ (b),(d),(f). Here, $\boldsymbol{E}$ is the best-fit linear strain tensor at $\boldsymbol{r}, \boldsymbol{E}^{\prime}$ is detraced $\boldsymbol{E}$, and $\boldsymbol{e}_{\mathrm{ext}}$ is a unit eigenvector corresponding to the largest eigenvalue of $\boldsymbol{E}$ at the origin. The radial axes are plotted on a $\log$ scale.

steady-state elasticity predicts $\hat{\gamma}_{\text {iso }, 0} \equiv 0$. Instead, we see a rapidly decaying radial wave of isotropic expansion and contraction, with expansion peaks (compression troughs) roughly coinciding with the peaks (troughs) of $g(r)$ [33]. This is consistent with the results of [44] in the context of athermal quasistatic shear: rearrangements expand the structure at the shortest distances from the rearrangement, thus locally hardening the structure, but compress the structure slightly further out, with this hardening-softening pattern repeating out to large distances.

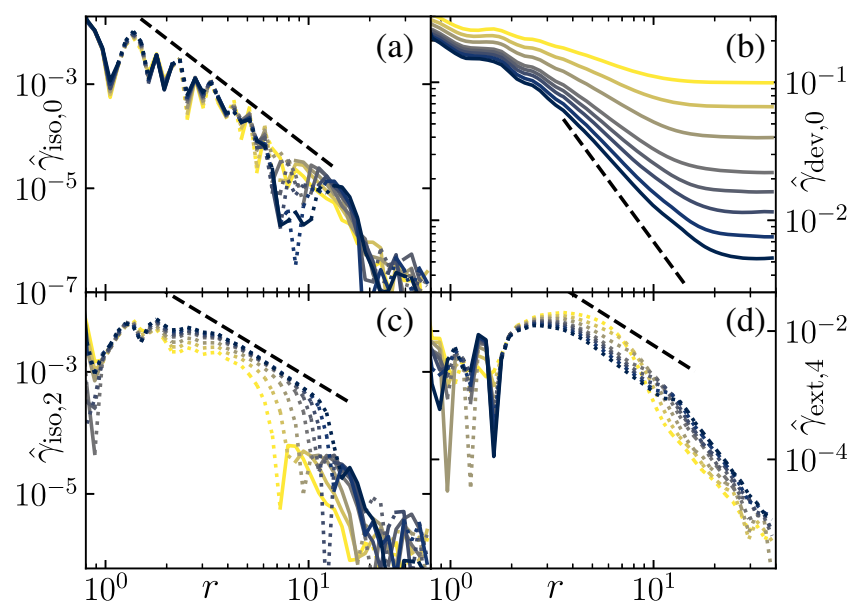

FIG. 2. (a)-(d) Fourier series cofficients $\hat{\gamma}_{\text {iso }, 0}, \hat{\gamma}_{\text {dev }, 0}, \hat{\gamma}_{\text {iso }, 2}$, and $\hat{\gamma}_{\text {ext } 4}$, respectively, as a function of distance $r$ from a rearranging particle (cf. Fig. 1). Dotted segments denote negative values. The colors correspond to temperatures $T=0.100,0.105,0.110$, $0.115,0.120,0.130,0.140$, and 0.150 (darkest to lightest). Dashed lines (b)-(d): slopes corresponding to the power-law decay, $\propto r^{-2}$, of an infinite elastic medium responding to a small plastic deformation. Dashed line (a): power law decay $\propto r^{-3}$ plotted as an upper bound to the envelope of $\hat{\gamma}_{\text {iso, }, 0}$.

We now focus directly on dynamic facilitation and analyze its relation with the elastic responses characterized above. We use the pair distribution of rearrangements, $g_{\text {hop }}(\boldsymbol{r})=g_{\text {hop }}(r, \theta)$ (the average density of rearrangements at $\boldsymbol{r}$ given a rearrangement at the origin, divided by the bulk rearrangement density [33]), to investigate the extent to which facilitation of rearrangements is due to elasticity. In Figs. 3(a) and 3(b) we display $\tilde{g}_{\text {hop }}(r, \theta)=$ $2 \pi g_{\text {hop }}(r, \theta) / \int_{0}^{2 \pi} g_{\text {hop }}(r, \theta) d \theta$ [the rearrangement pair distribution $g_{\mathrm{hop}}(r, \theta)$ normalized at each $r$ by its angular mean $\left.(2 \pi)^{-1} \int_{0}^{2 \pi} g_{\text {hop }}(r, \theta) d \theta\right]$ for rearrangements over the time interval $[0, \Delta t][45]$ at distances $r=1,3,10,30$, for temperatures $T=0.100$ and $T=0.150$. Well below $T_{\mathrm{MCT}}$, at $T=0.100$ [Fig. 3(a)], the spatial distribution of subsequent rearrangements remains anisotropic out to $r=30$. The angular Fourier series expansion coefficients of $\tilde{g}_{\text {hop }}$ [33] show that this anisotropy comes from the second- and fourth-order Fourier modes of $\tilde{g}_{\text {hop }}$, and persists up to at least $r \approx 20$. Above $T_{\mathrm{MCT}}$, at $T=0.150$ [Fig. 3(b)], no anisostropic structure can be discerned beyond the second neighbor shell, i.e., beyond $r>3$.

The anisotropy in $g_{\text {hop }}$ upon coaligning the local strain tensors of plastic events is an unambiguous measure of the elastic component of $g_{\text {hop }}$. To quantify the amount of anisotropy in the spatial distribution of rearrangements we introduce the mean squared anisotropy (deviation from isotropy) $\alpha(r)=(2 \pi)^{-1} \int_{0}^{2 \pi}\left[\tilde{g}_{\text {hop }}(r, \theta)-1\right]^{2} d \theta$, as shown in Fig. 3(c). When calculating $\alpha$ in practice, we control for the effect of statistical noise, which is larger at lower 

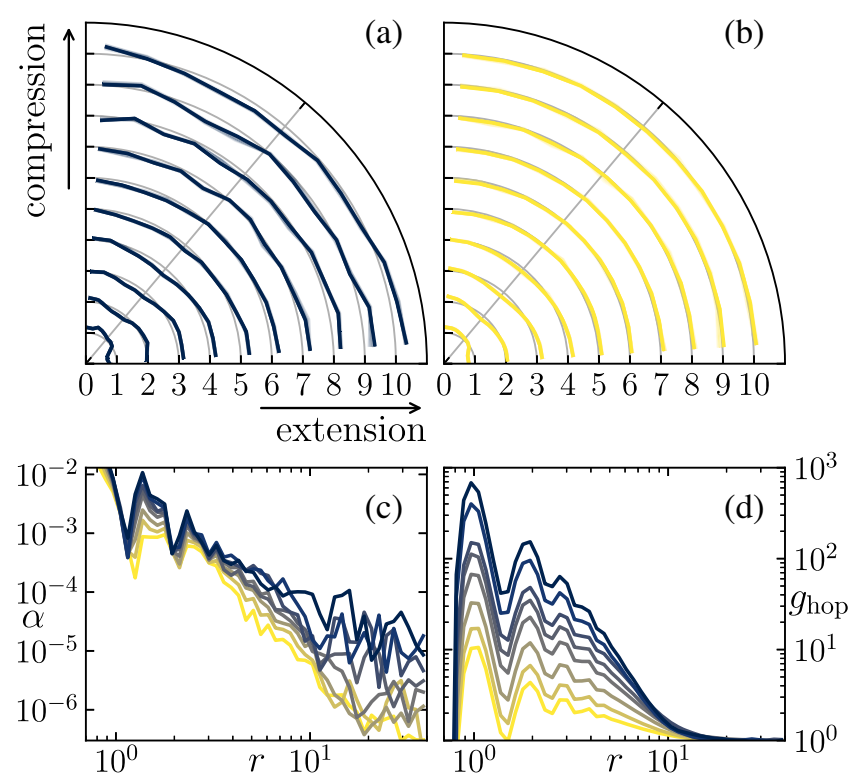

FIG. 3. Top row: plots of the normalized pair distribution of rearrangements $\tilde{g}_{\text {hop }}=2 \pi g_{\text {hop }} / \int_{0}^{2 \pi} g_{\text {hop }} d \theta$ across one rearrangement interval $\Delta t=10^{2}$ for temperatures (a) $T=0.100$ and (b) 0.150 . Each curve corresponds to a different $r$, and is scaled so that isotropy (dashed black circle) corresponds to the corresponding $r$ value for the log-scaled $r$ of Fig. 1. (c) Mean squared anisotropy $\alpha(r)=(2 \pi)^{-1} \int_{0}^{2 \pi}\left(\tilde{g}_{\text {hop }}-1\right)^{2} d \theta$ as a function of $r$ for $T=0.100,0.105,0.110,0.115,0.120,0.130,0.140$, and 0.150 (lighter shade denoting higher $T$ ). Transparent curves: results of bootstrap sampling (sampling hops with replacement from the set of hops in the raw data, while preserving the number of hops per $r$-bin) to indicate the statistical uncertainty of the results. (d): Pair correlation of hops $g_{\text {hop }}(r)$, same color coding as (c).

temperatures due to these temperatures having fewer rearrangements per trajectory, as described in [33]. High $\alpha$ at small $r$ shows that displacements at this scale, though highly nonlinear, are measurably influenced by the orientation of the linear strain. This short-ranged influenceindicative of short-ranged dynamic facilitation-persists over the entire temperature range studied. As $T$ is lowered, the large- $r$ tail of $\alpha$ increases markedly, spanning two orders of magnitude, implying a significant difference in the role played by long-range elastic interactions between the lowest and highest temperatures considered here. At the same time, Fig. 3(d) shows that hops become more strongly correlated with decreasing temperature, with the dynamic facilitation implied by correlations $g_{\text {hop }}>1$ surviving up to increasingly large distances as a result.

To quantify the extent of elastically mediated dynamic facilitation, we introduce $I_{\alpha}:=\int_{2.5}^{40} \alpha(r) d r$, which integrates $\alpha$ over distances beyond the second neighbor shell at $r \gtrsim 2.5$ (to filter out the effect of short-range facilitation) and up to the limit beyond which finite-size effects appear. Figure 4(a) displays its evolution with $1 / T$. We observe a crossover at $T_{\mathrm{MCT}}$ from a slow to a rapid growth of $I_{\alpha}$ as a function of $1 / T$. Above $T_{\mathrm{MCT}}$, the strain-correlated spatial

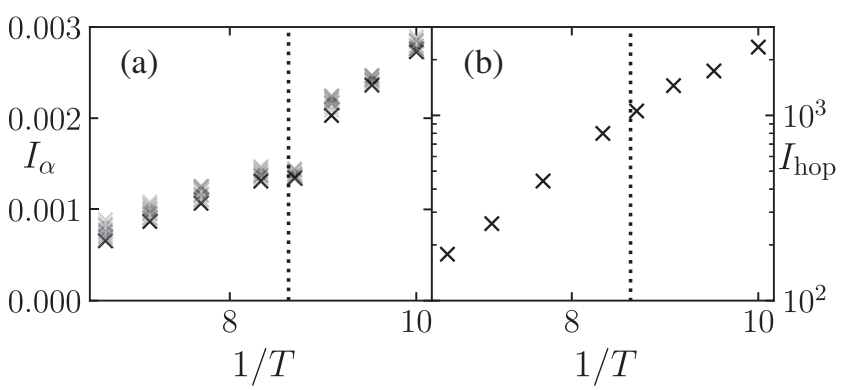

FIG. 4. Integrated (a) mean squared anisotropy $I_{\alpha}=\int_{2.5}^{40} \alpha(r) d r$ and (b) pair distribution of rearrangements $I_{\text {hop }}=\int_{2.5}^{40} 2 \pi r\left[g_{\text {hop }}(r)-1\right] d r$ as a function of inverse temperature $1 / T$. Transparent crosses in (a) are the results of bootstrap sampling, which accentuates fluctuations. Dotted lines: $1 / T_{\mathrm{MCT}}$.

organization of the rearrangements is largely obscured, beyond the second-neighbor shell, by other rearrangements [33]. The competition between these other rearrangements and emergent elasticity at longer distances leads to the observed crossover in $I_{\alpha}$. Note that the observed crossover at $T_{\mathrm{MCT}}$ is robust to choices of lower bound of the integral, rearrangement interval $\Delta t$, strain length $\xi_{\mathrm{FL}}$, and starting point for the integration $\left(r_{0} \geq 2.5\right)$ [33]. We similarly define an integrated excess density of rearrangements (relative to the bulk rearrangement density), $I_{\text {hop }}:=\int_{2.5}^{40} 2 \pi r\left[g_{\text {hop }}(r)-1\right] d r$, where $g_{\text {hop }}(r)=$ $(2 \pi)^{-1} \int_{0}^{2 \pi} g_{\text {hop }}(r, \theta) d \theta$. Figure $4\left(\right.$ b) shows that $I_{\text {hop }}$, quantifying the strength of correlations between hops, and hence dynamic facilitation, increases with decreasing $T$. Taken together, these results for $g_{\text {hop }}, I_{\alpha}$, and $I_{\text {hop }}$ provide direct and explicit evidence for the elastoplastic mechanism, in which the elastic response to a rearrangement triggers other, distant rearrangements. The elastoplastic mechanism emerges as a significant component of an increasingly prominent facilitation upon crossing $T_{\mathrm{MCT}}$.

Taken together, our findings suggest a crossover from solely short-ranged dynamic facilitation for $T>T_{\mathrm{MCT}}$ to additional long-ranged, elastically mediated dynamic facilitation of avalanches at $T<T_{\text {MCT }}$. This finding provides an intriguing link between mean-field models, where $T_{\mathrm{MCT}}$ marks the location where supercooled liquids first exhibit a transient solidlike behavior, and real-space dynamical heterogeneity. We note that the crossover at $T_{\mathrm{MCT}}$ is not visible in the strain field induced by a rearrangement; for example, the isotropic strain shows dipolar angular behavior at all temperatures considered. The crossover is only evident in the spatial and angular distribution of rearrangements that follow soon after the original rearrangement at the origin. The anisotropic spatial distribution of hops evidenced in $g_{\text {hop }}$, Figs. 3(a), 3(b), and the increase in range of $g_{\text {hop }}(r)$, Fig. 3(d), as well as the crossover at $T=T_{\text {MCT }}$ seen in $I_{\alpha}$, Fig. 4(a), can only be due to the strain field created by the rearrangement at the origin. At high $T$ strain does not facilitate rearrangements because the 
rearrangements are so abundant and closely spaced that their strain fields effectively interfere [33]. We note that at temperatures below $T_{\mathrm{MCT}}$, dynamical heterogeneity still involves short-ranged facilitation, whereby the local relaxation of clusters coalesces on longer length scales to form avalanches. Nevertheless, the emergence of prominent long-ranged elastic effects must influence the spatiotemporal correlations between such avalanches.

Approximately ten years ago it was noticed that rare, long-ranged "surges" of dynamical heterogeneity connected to strain deformation occur in supercooled liquids [46], and that such behavior occurs in some coarse-grained kinetically constrained models [3,5,47]. Our findings potentially pinpoint the microscopic underpinnings of such behavior, which could lead to greater understanding of the building blocks of phenomenological models of supercooled liquids, though further work is needed to confirm this crossover in other systems, particularly experimental or three-dimensional systems. It would also be worthwhile to generalize elastoplastic models [16] to describe elastic dynamic facilitation within equilibrium thermal dynamics along the lines of $[48,49]$. More generally, our results highlight the importance of long-ranged elastic processes in mediating the organization of dynamical heterogeneities on long length and timescales, and the need to incorporate such processes in simplified models of the glass transition. In light of recent work [50,51] showing evidence that elasticity sets the activation energy barriers to rearrangement of deeply supercooled liquids, and hence their short timescale dynamics [52], it is tempting to conclude that elastoplasticity alone can account for most of the dynamics of supercooled liquids below $T_{\mathrm{MCT}}$.

Data sets and code for the work in this paper can be found at Ref. [53].

We thank M. Ozawa and L. Berthier for providing us with equilibrated configurations and the code to generate them, C. Scalliet for providing the swap potential files for use with LAMMPS, and E. Corwin for sharing computational resources with us in the early stages of this project. R. N. C. thanks S. Ridout, G. Zhang, and I. Tah for discussions. This work received funding from the Simons Collaboration "Cracking the glass problem" via Grants No. 454935 (G. B.), No. 454945 (A. J. L.), No. 454951 (D. R. R.), and No. 348126 (R. N. C.), and from a Simons Investigator grant (Grant No. 327939 to A. J. L.). This work used the Extreme Science and Engineering Discovery Environment (XSEDE) [54], which is supported by National Science Foundation Grant No. ACI-1548562.

*Corresponding author. chackorn@sas.upenn.edu

[1] L. Berthier, G. Biroli, J.-P. Bouchaud, L. Cipelletti, and W. van Saarloos, Dynamical Heterogeneities in Glasses,
Colloids, and Granular Media (Oxford University Press, Oxford, 2011), Vol. 150.

[2] G. H. Fredrickson and H. C. Andersen, Kinetic Ising Model of the Glass Transition, Phys. Rev. Lett. 53, 1244 (1984).

[3] D. Chandler and J. P. Garrahan, Dynamics on the way to forming glass: Bubbles in space-time, Annu. Rev. Phys. Chem. 61, 191 (2010).

[4] R. Candelier, A. Widmer-Cooper, J. K. Kummerfeld, O. Dauchot, G. Biroli, P. Harrowell, and D. R. Reichman, Spatiotemporal Hierarchy of Relaxation Events, Dynamical Heterogeneities, and Structural Reorganization in a Supercooled Liquid, Phys. Rev. Lett. 105, 135702 (2010).

[5] A. S. Keys, L. O. Hedges, J. P. Garrahan, S. C. Glotzer, and D. Chandler, Excitations are Localized and Relaxation is Hierarchical in Glass-Forming Liquids, Phys. Rev. X 1, 021013 (2011).

[6] Y.S. Elmatad and A. S. Keys, Manifestations of dynamical facilitation in glassy materials, Phys. Rev. E 85, 061502 (2012).

[7] P. W. Anderson, B. I. Halperin, and C. M. Varma, Anomalous low-temperature thermal properties of glasses and spin glasses, Philos. Mag. 25, 1 (1972).

[8] W. A. Phillips, Tunneling states in amorphous solids, J. Low Temp. Phys. 7, 351 (1972).

[9] J. Joffrin and A. Levelut, Virtual phonon exchange in glasses, J. Phys. II (France) 36, 811 (1975).

[10] G. Picard, A. Ajdari, F. Lequeux, and F. Bocquet, Elastic consequences of a single plastic event: A step towards the microscopic modeling of the flow of yield stress fluids, Eur. Phys. J. E 15, 371 (2004).

[11] K. Martens, L. Bocquet, and J.-L. Barrat, Connecting Diffusion and Dynamical Heterogeneities in Actively Deformed Amorphous Systems, Phys. Rev. Lett. 106, 156001 (2011).

[12] R. Dasgupta, S. Karmakar, and I. Procaccia, Universality of the Plastic Instability in Strained Amorphous Solids, Phys. Rev. Lett. 108, 075701 (2012).

[13] O. Gendelman, P. K. Jaiswal, I. Procaccia, B. S. Gupta, and J. Zylberg, Shear transformation zones: State determined or protocol dependent? Europhys. Lett. 109, 16002 (2015).

[14] S. Patinet, D. Vandembroucq, and M. L. Falk, Connecting Local Yield Stresses with Plastic Activity in Amorphous Solids, Phys. Rev. Lett. 117, 045501 (2016).

[15] Z. Budrikis, D. F. Castellanos, S. Sandfeld, M. Zaiser, and S. Zapperi, Universal features of amorphous plasticity, Nat. Commun. 8, 15928 (2017).

[16] A. Nicolas, E. E. Ferrero, K. Martens, and J.-L. Barrat, Deformation and flow of amorphous solids: Insights from elastoplastic models, Rev. Mod. Phys. 90, 045006 (2018).

[17] A. Barbot, M. Lerbinger, A. Lemaître, D. Vandembroucq, and S. Patinet, Rejuvenation and shear banding in model amorphous solids, Phys. Rev. E 101, 033001 (2020).

[18] V. A. Levashov, J. R. Morris, and T. Egami, Viscosity, Shear Waves, and Atomic-Level Stress-Stress Correlations, Phys. Rev. Lett. 106, 115703 (2011).

[19] K. E. Jensen, D. A. Weitz, and F. Spaepen, Local Shear Transformations in Deformed and Quiescent Hard-Sphere Colloidal Glasses, Phys. Rev. Lett. 90, 042305 (2014).

[20] A. Lemaître, Structural Relaxation is a Scale-Free Process, Phys. Rev. Lett. 113, 245702 (2014). 
[21] A. Lemaître, Tensorial analysis of eshelby stresses in 3D supercooled liquids, J. Chem. Phys. 143, 164515 (2015).

[22] B. Wu, T. Iwashita, and T. Egami, Anisotropic stress correlations in two-dimensional liquids, Phys. Rev. E 91, 032301 (2015).

[23] B. Illing, S. Fritschi, D. Hajnal, C. Klix, P. Keim, and M. Fuchs, Strain Pattern in Supercooled Liquids, Phys. Rev. Lett. 117, 208002 (2016).

[24] A. Lemaître, Inherent stress correlations in a quiescent twodimensional liquid: Static analysis including finite-size effects, Phys. Rev. E 96, 052101 (2017).

[25] A. Lemaître, Stress correlations in glasses, J. Chem. Phys. 149, 104107 (2018).

[26] J. P. Garrahan, P. Sollich, and C. Toninelli, Kinetically constrained models, in Dynamical Heterogeneities in Glasses, Colloids, and Granular Media, International Series of Monographs on Physics, edited by L. Berthier, G. Biroli, J.-P. Bouchaud, L. Cipelletti, and W. van Saarloos (Oxford University Press, Oxford, 2011), pp. 341-369.

[27] G. Biroli and J.-P. Bouchaud, The random first-order transition theory of glasses: A critical assessment, in Structural Glasses and Supercooled Liquids: Theory, Experiment, and Applications, edited by P. G. Wolynes and V. Lubchenkos (Wiley, New York, 2012), pp. 31-113.

[28] M. Ozawa, L. Berthier, G. Biroli, and G. Tarjus, Role of fluctuations in the yielding transition of two-dimensional glasses, Phys. Rev. Research 2, 023203 (2020).

[29] S. Plimpton, Fast parallel algorithms for short-range molecular dynamics, J. Comp. Phys. 117, 1 (1995).

[30] L. Berthier, P. Charbonneau, A. Ninarello, M. Ozawa, and S. Yaida, Zero-temperature glass transition in two dimensions, Nat. Commun. 10, 1508 (2019).

[31] L. Berthier, D. Coslovich, A. Ninarello, and M. Ozawa, Equilibrium Sampling of Hard Spheres up to the Jamming Density and Beyond, Phys. Rev. Lett. 116, 238002 (2016).

[32] A. Ninarello, L. Berthier, and D. Coslovich, Models and Algorithms for the Next Generation of Glass Transition Studies, Phys. Rev. X 7, 021039 (2017).

[33] See the Supplemental Material at http://link.aps.org/ supplemental/10.1103/PhysRevLett.127.048002 for additional detail on our methodologies, which includes Refs. [34-42].

[34] E. Flenner and G. Szamel, Fundamental differences between glassy dynamics in two and three dimensions, Nat. Commun. 6, 7392 (2015).

[35] S. Vivek, C. P. Kelleher, P. M. Chaikin, and E. R. Weeks, Long-wavelength fluctuations and the glass transition in two dimensions and three dimensions, Proc. Natl. Acad. Sci. U.S.A. 114, 1850 (2017).

[36] B. Illing, S. Fritschi, H. Kaiser, C. L. Klix, G. Maret, and P. Keim, Mermin-Wagner fluctuations in 2D amorphous solids, Proc. Natl. Acad. Sci. U.S.A. 114, 1856 (2017).

[37] R. Das, I. Tah, and S. Karmakar, Possible universal relation between short time $\alpha$-relaxation and long time $\beta$-relaxation in glass-forming liquids, J. Chem. Phys. 149, 024501 (2018).
[38] I. Tah, S. Sengupta, S. Sastry, C. Dasgupta, and S. Karmakar, Glass Transition in Supercooled Liquids with Medium-Range Crystalline Order, Phys. Rev. Lett. 121, 085703 (2018).

[39] N. Lačević, F. W. Starr, T. B. Schrøder, and S. C. Glotzer, Spatially heterogeneous dynamics investigated via a timedependent four-point density correlation function, J. Chem. Phys. 119, 7372 (2003).

[40] E. D. Cubuk et al., Structure-property relationships from universal signatures of plasticity in disordered solids, Science 358, 1033 (2017).

[41] B. Efron and R. Tibshirani, Bootstrap methods for standard errors, confidence intervals, and other measures of statistical accuracy, Stat. Sci. 1, 54 (1986).

[42] S. Chandrasekhar, Stochastic problems in physics and astronomy, Rev. Mod. Phys. 15, 1 (1943).

[43] M. L. Falk and J. S. Langer, Dynamics of viscoplastic deformation in amorphous solids, Phys. Rev. E 57, 7192 (1998).

[44] G. Zhang, S. Ridout, and A. J. Liu, Interplay of rearrangements, strain, and local structure during avalanche propagation, arXiv:2009.11414.

[45] By considering the same time interval for all rearrangements, we cannot tell, given a pair of rearrangements, which rearrangement triggered the other. However, time reversibility means that this causal ambiguity exists even for events that are well separated in time. We have checked [33] that choosing pairs of events in separate time intervals does not qualitatively affect our results.

[46] A. Widmer-Cooper and P. Harrowell, Central role of thermal collective strain in the relaxation of structure in a supercooled liquid, Phys. Rev. E 80, 061501 (2009).

[47] The nature of "surges" in the kinetically constrained East model is discussed in [6].

[48] M. Popović, T. W. de Geus, W. Ji, and M. Wyart, Thermally activated flow in models of amorphous solids, arXiv:2009.04963.

[49] J. T. Parley, S. M. Fielding, and P. Sollich, Aging in a mean field elastoplastic model of amorphous solids, Phys. Fluids 32, 127104 (2020).

[50] M. R. Hasyim and K. K. Mandadapu, A theory of localized excitations in supercooled liquids, arXiv:2103.03015.

[51] G. Kapteijns, D. Richard, E. Bouchbinder, T. B. Schrøder, J.C. Dyre, and E. Lerner, Does mesoscopic elasticity control viscous slowing down in glassforming liquids? arXiv:2103.11404.

[52] B. Guiselin, C. Scalliet, and L. Berthier, Microscopic origin of excess wings in relaxation spectra of deeply supercooled liquids, arXiv:2103.01569.

[53] https://figshare.com/articles/dataset/_/14943204 and https:// bitbucket.org/chackorn/elastoplasticity-mediates-dynamical.

[54] J. Towns, T. Cockerill, M. Dahan, I. Foster, K. Gaither, A. Grimshaw, V. Hazlewood, S. Lathrop, D. Lifka, G. D. Peterson, R. Roskies, J. R. Scott, and N. Wilkins-Diehr, XSEDE: Accelerating scientific discovery, Computing in Science \& Engineering 16, 62 (2014). 